\title{
The Impact of Valid Pedometer Days on Average Daily Steps and Wear Time in Children
}

\author{
Natalie E. Houser ${ }^{1, *}$, Scott W. Donald ${ }^{2}$, Angela M. Kolen ${ }^{3}$ \\ ${ }^{1}$ College of Kinesiology, University of Saskatchewan, Saskatoon, Canada \\ ${ }^{2}$ Department of Kinesiology, Wilfred Laurier University, Waterloo, Canada \\ ${ }^{3}$ Department of Human Kinetics, St. Francis Xavier University, Antigonish, Canada \\ *Corresponding author: natalie.houser@usask.ca \\ Received April 11, 2019; Revised May 17, 2019; Accepted June 15, 2019
}

\begin{abstract}
Pedometers reliably measure physical activity with established guidelines regarding wear time for hours per day, days per week, and minimum and maximum steps to provide sufficient data. This paper examined children in grades 3 to 6 for potential differences in average steps and average wear time according to the number of days of pedometer data with at least 10 hours of self-reported wear time and steps between 1000 and 30,000. As part of the Canadian Assessment of Physical Literacy, physical activity data for a maximum of seven consecutive days were obtained from 852 (girls $n=465$; boys $n=387$ ) children using Piezo RX Steps Count pedometers. Average daily step count was $11,767 \pm 3,284$, slightly less than recommended; average self-reported wear time was $12.9 \pm 0.9$ hours. One-way ANOVAs demonstrated significant differences in average daily steps and average daily wear time between girls and boys and between grades. ANOVAs also examined differences in daily steps and wear time of the children when grouped according to valid days of pedometer data (1-2, 3-4, 5-6, and 7 days). In general, younger children (i.e., grades $3 \& 4$ ) wore the pedometer for less time than older children (i.e., grades $5 \& 6$ ). Given the significant differences in steps according to pedometer wear time found in this study, we may not be obtaining a thorough understanding of children's physical activity behaviours. It may be helpful to include children with fewer than three days of pedometer wear time data to provide a better understanding of children's physical activity levels as a whole and in particular for promoting physical activity for boys girls in varying grades.
\end{abstract}

Keywords: boys and girls, physical activity, measurement, pedometer criteria

Cite This Article: Natalie E. Houser, Scott W. Donald, and Angela M. Kolen, "The Impact of Valid Pedometer Days on Average Daily Steps and Wear Time in Children." Journal of Physical Activity Research, vol. 4, no. 2 (2019): 103-107. doi: 10.12691/jpar-4-2-4.

\section{Introduction}

A common tool currently used to quantify participation in physical activity, and in particular adherence to physical activity guidelines, is pedometers. Pedometers are considered valid and reliable measurement tools for children's physical activity [1], providing total daily steps, as well as the number of steps at a moderate or vigorous level of intensity. Canadian school-aged children are recommended to obtain a minimum of 60 minutes of moderate-to-vigorous physical activity (MVPA) each day as part of the physical activity recommendations included in the 24-hour movement guidelines [2]. This recommended 60 minutes of MVPA is equivalent to approximately 12,000 steps per day [3]. Only $35 \%$ of Canadian children aged 5 to 17 years obtain an average of 12,000 steps per day [4].

In attempts to learn about population levels of physical activity and sedentary behaviours, it is important to consider how physical activity is measured, including what constitutes valid and reliable data. Further, the differences that exist between boys and girls and among children according to age or grade should be considered. Colley et al. noted that Canadian boys obtained more steps than girls with an average of 12,100 versus 10,300 steps [5]. Similar findings were found internationally, where average daily steps among children in grades 4 to 6 were significantly lower in girls than boys, with 9,545 and 12,303 steps respectively [6]. Given these differences between boys and girls, it may be useful to learn more about pedometer wear time (hours per day and the number of days worn) and how it might vary between boys and girls and whether these potential differences influence the data collection process, the data obtained, and our understanding of their level of physical activity. Differences in average daily steps according to age (or grade) have also been reported, as physical activity levels decrease with increasing age/grade in boys and girls [7]. A different study identified declines in physical activity over 12 months, with the percentage of children meeting the physical activity guidelines decreasing by approximately five percent [8]. Additionally, Corder et al. [8] identified that physical activity was more likely to decrease in girls than boys. Growth, maturation, and development likely 
play a substantive role in these decreases in physical activity and the differences between boys and girls [8,9].

Current guidelines regarding the collection and use of pedometer data with children as a measure of their overall physical activity suggest at least three days of wear time are needed [10]. Further, a valid day of pedometer data must include at least 10 hours of wear time [11,12] and step counts between 1000 and 30,000 [10,13]. In general, when these standards have not been met participants' data are removed from further analyses. The potential differences that may exist among children obtaining three or more days of valid pedometer data compared to those with one to two days of valid data requires further exploration. It is possible that better understanding of children who provide less physical activity data in research studies may help in our physical activity promotion efforts overall. As such, this study explores the application of the 'minimum three days of valid pedometer data' guideline in a relatively large crosssectional study with pedometer data from children in grades 3 to 6 (aged 7 to 12 years). Additionally, pedometer wear time and average daily steps were compared between boys and girl and among boys and girls in grades 3 to 6 .

\section{Methods}

\subsection{Participants}

Participants in this study were part of the Canadian Assessment of Physical Literacy (CAPL) project (https://www.capl-ecsfp.ca). A convenience sample of elementary school students from ten schools within northeastern Nova Scotia, Canada were recruited to participate in CAPL. Children in grades 3 to 6 present on the day of recruitment were invited to participate via a verbal introduction and explanation of the study and formally through a letter of invitation for their parents/guardians. Parental/guardian written informed consent and complete health record checks were obtained for 1065 children. Children provided verbal assent at the time of data collection. Research ethics approval was obtained by a children's hospital, the local university, and the relevant school board.

\subsection{Physical Activity Measurements}

Piezo RX Steps Count pedometers were provided to participating children as part of the CAPL protocol to obtain an estimate of their daily physical activity. These children were instructed to wear the pedometer over their right hip on their waistband all day for seven consecutive days (not counting the day of distribution). Children were also asked to record the time they put the pedometer on in the morning and the time they took it off at night in a pedometer tracking log. Further, participants were asked to record any time they took the pedometer off and the reason for non-wear time. A follow up email sent to parents/guardians of the children explained this protocol for their children. The day children received the pedometers was considered a "practice day" with the seven days of wear time beginning the following day.
Following previously established protocols, a valid day of pedometer data were obtained when step counts were between 1000 and 30,000 steps per day [10,13] and the self-report $\log$ indicated the pedometer was worn for at least 10 hours $[11,12]$. The children whose data met these requirements were grouped according to 0 days, 1-2 days, 3-4 days, 5-6 days, or 7 days of pedometer data for further analyses.

\subsection{Data Analysis}

Descriptive statistics were used to determine the mean and standard deviation of steps per day and daily wear time for the children with at least 10 hours of self-reported wear time and between 1000 and 30,000 steps and 1-2 days, 3-4 days, 5-6 days, and 7 days of pedometer data. Using Statistical Package for Social Sciences (SPSS, V.24) and one-way analyses of variance (ANOVA) the average daily steps and average daily wear time for the children were compared between these groups (i.e., 1-2 days, 3-4 days, 5-6 days, or 7 days). Further analyses examined grade $(3,4,5, \& 6)$ and sex differences in average daily steps and average daily wear time overall (regardless of number of valid days).

\section{Results}

Of the 1065 children who provided verbal assent and parental/guardian consent to participate in the CAPL project, 156 returned pedometers without data other than the practice day. As such, pedometer data from 909 children or $85.4 \%$ of the original sample were available for further analyses. Of these 909 children, 852 (465 girls; 387 boys) provided one or more days of pedometer data with at least 10 hours of self-reported wear time and step counts between 1000 and 30,000. Table 1 shows the dispersion of this sample according to grade and sex. Although $48.4 \%$ of the CAPL sample were boys, fewer (i.e., $45.4 \%$ ) provided at least one day of valid pedometer data. Of the children $(\mathrm{n}=212)$ that did not provide pedometer data (either no data $(n=156)$ or no days with at least 10 hours of self-recorded wear time and/or steps between 1000 and 30,000 $(n=56)), 60.4 \%$ were boys.

Table 1. Boys and girls in grades $3,4,5$, \& 6 with $0,1-2,3-4,5-6$, or 7 days valid pedometer data*

\begin{tabular}{|c|c|c|c|c|c|c|c|c|}
\hline & \multicolumn{4}{|c|}{ Girls } & \multicolumn{4}{c|}{ Boys } \\
\hline Days/Grade & 3 & 4 & 5 & 6 & 3 & 4 & 5 & 6 \\
\hline $\mathbf{0}$ & 2 & 12 & 3 & 1 & 4 & 25 & 5 & 4 \\
\hline $\mathbf{1 - 2}$ & 2 & 7 & 3 & 8 & 2 & 13 & 2 & 1 \\
\hline $\mathbf{3 - 4}$ & 10 & 39 & 20 & 17 & 8 & 54 & 14 & 17 \\
\hline $\mathbf{5 - 6}$ & 30 & 94 & 45 & 55 & 24 & 72 & 26 & 42 \\
\hline $\mathbf{7}$ & 12 & 63 & 30 & 31 & 17 & 39 & 26 & 30 \\
\hline Total & $\mathbf{5 6}$ & $\mathbf{2 1 5}$ & $\mathbf{1 0 1}$ & $\mathbf{1 1 2}$ & $\mathbf{5 5}$ & $\mathbf{2 0 3}$ & $\mathbf{7 3}$ & $\mathbf{9 4}$ \\
\hline
\end{tabular}

* valid pedometer data $=$ at least 10 hours and steps $\geq 1,000$ and $\leq 30,000$.

A closer examination of the invalid pedometer data indicates that of the 6363 pedometer days possible (i.e., 909 x 7 days), 96 days (1.5\%) were excluded because of an invalid count; $91 / 96<1000$ steps; 5/96 > 30,000 steps. Incomplete, incorrectly completed, or not returned pedometer 
logs excluded $922(14.5 \%)$ pedometer days. Interestingly, $718(11.3 \%)$ of potential pedometer days recorded in the $\operatorname{logs}$ with sufficient reported wear time were excluded because there were no steps on the pedometer for those days or the pedometer was not returned. Finally, 761 $(12.0 \%)$ pedometer days were not included because of insufficient self-reported wear time, i.e., $<10$ hours.

Table 2 identifies the average $( \pm$ SD) pedometer wear time for the boys and girls separately in grades 3, 4, 5 and 6. The girls in grade 6 and boys in grade 4 with 1-2 days of pedometer data wore the pedometer less than their counterparts with 5-6 or 7 days of pedometer data. Further, girls in grade 4 with 3-4 days of pedometer data wore the pedometer for less time than the girls who provided 5-6 or 7 days. Lastly, boys in grade 3 with 5-6 days of pedometer data wore the pedometer less than the group that provided 7 days of data.

The average daily step count for the combined sample of children (boys and girls; grades 3-6) with at least one day of pedometer data with minimally 10 hours self-reported wear time and steps between 1000 and 30,000 is $11,767 \pm 3,284$. Table 3 presents average $( \pm$ SD) steps per day for the total sample according to number of days of pedometer data. A significant difference in the average daily steps children obtained depending on valid pedometer days of data were found $(\mathrm{F}(3,848)=3.32$, $\mathrm{p}=0.019$ ) with post hoc analyses showing the children with 7 days data obtained significantly more steps than those with 1-2 days of data.

Further analyses explored the average steps per day according to grade and sex. No significant differences were found in these analyses except in the ANOVA for the boys in grade 4. However, post-hoc analysis of this data $(p=0.057)$ did not indicate specific significant differences. As such, there were no significant differences in the average steps across grades in boys or girls according to the number of days of valid pedometer data, as seen in Table 4.

Figure 1 and Figure 2 present the average daily wear time and steps for boys and girls by grade respectively. Analysis of average daily wear time for girls identified a significant difference existed among the grades $(\mathrm{F}(3,461)=5.34, \mathrm{p}=0.001)$ with post hoc analyses showing the girls in grade 3 wore the pedometer significantly less than the girls in grades 5 and 6 (see Figure 1). For boys, there was also a significant difference in average daily wear time between grades $(F(3,383)=10.8, p=0.000)$ with post hoc analyses showing the boys in grade 3 and 4 wore the pedometer less than the boys in grades 5 (see Figure 1).

Table 2. Average ( \pm SD) pedometer wear time (hours) for boys and girls in grades 3, 4, 5, \& 6 according to 1-2, 3-4, 5-6, or 7 valid days of pedometer data

\begin{tabular}{|c|c|c|c|c|c|c|c|c|}
\hline & \multicolumn{4}{|c|}{ Girls } & \multicolumn{4}{c|}{ Boys } \\
\hline Days/Grade & $\mathbf{3}$ & $\mathbf{4}$ & $\mathbf{5}$ & $\mathbf{6}$ & $\mathbf{3}$ & $\mathbf{4}$ & $\mathbf{5}$ & $\mathbf{6}$ \\
\hline $\mathbf{1 - 2}$ & $12.1 \pm 2.0$ & $12.8 \pm 0.6$ & $13.4 \pm 0.5$ & $12.2 \pm 0.7$ & $12.4 \pm 0.6$ & $12.1 \pm 1.0$ & $13.5 \pm 0.5$ & $13.5^{\wedge}$ \\
\hline $\mathbf{3 - 4}$ & $12.2 \pm 1.2$ & $12.4 \pm 0.8$ & $12.8 \pm 1.1$ & $12.7 \pm 1.1$ & $12.4 \pm 0.9$ & $12.6 \pm 1.0$ & $13.3 \pm 1.6$ & $12.9 \pm 1.0$ \\
\hline $\mathbf{5 - 6}$ & $12.7 \pm 0.8$ & $12.9 \pm 0.9^{* *}$ & $13.0 \pm 0.8$ & $13.2 \pm 0.8^{*}$ & $12.5 \pm 0.6$ & $12.8 \pm 0.9^{*}$ & $12.9 \pm 0.7$ & $13.3 \pm 0.8$ \\
\hline $\mathbf{7}$ & $12.7 \pm 0.5$ & $13.0 \pm 0.7^{* *}$ & $13.4 \pm 0.7$ & $13.2 \pm 0.8^{*}$ & $13.1 \pm 0.6 * * *$ & $13.0 \pm 0.8^{*}$ & $13.4 \pm 0.7$ & $13.5 \pm 1.0$ \\
\hline
\end{tabular}

* significantly different from $1-2$ days $(\mathrm{p}<0.05)$

** significantly different from $3-4$ days $(\mathrm{p}<0.05)$

$* * *$ significantly different from $5-6$ days $(\mathrm{p}<0.05)$

$\wedge$ one participant, therefore no standard deviation.

Table 3. Overall (i.e., boys and girls; in grades $3,4,5, \&$ 6) average $( \pm$ SD) steps per day according to valid pedometer days

\begin{tabular}{|c|c|c|c|c|c|c|}
\hline Days & $\mathbf{1 - 2}$ & $\mathbf{3 - 4}$ & $\mathbf{5 - 6}$ & $\mathbf{7}$ & $\mathbf{F}$ & $\mathbf{p}$ \\
\hline Steps & $10,737 \pm 3,448$ & $11,335 \pm 3,532$ & $11,834 \pm 3,174$ & $12,128 \pm 3,197$ & 3.323 & $0.019^{*}$ \\
\hline
\end{tabular}

* significant at $\mathrm{p}<0.05$.

Table 4. Average ( \pm SD) daily steps for boys and girls in grades (gr) 3, 4, 5, \& 6 according to valid pedometer days

\begin{tabular}{|c|c|c|c|c|c|c|c|}
\hline Sex & Gr & $\begin{array}{c}\begin{array}{c}1-2 \text { days } \\
\mathbf{n}=37\end{array} \\
\end{array}$ & $\begin{array}{c}\begin{array}{c}\text { 3-4 days } \\
n=179\end{array} \\
\end{array}$ & $\begin{array}{c}\text { 5-6 days } \\
n=388\end{array}$ & $\begin{array}{l}7 \text { days } \\
\mathrm{n}=248\end{array}$ & $\mathbf{F}$ & $\mathbf{p}$ \\
\hline \multirow{4}{*}{ Girls } & 3 & $13,923 \pm 1,755$ & $11,804 \pm 2,729$ & $13,205 \pm 3,775$ & $12,668 \pm 2,964$ & 0.50 & 0.68 \\
\hline & 4 & $11,947 \pm 3,711$ & $11,038 \pm 3,084$ & $11,302 \pm 2,882$ & $11,766 \pm 2,911$ & 0.63 & 0.60 \\
\hline & 5 & $11,167 \pm 1,089$ & $9,975 \pm 2,933$ & $11,096 \pm 3,069$ & $11,007 \pm 2,922$ & 0.71 & 0.55 \\
\hline & 6 & $9,006 \pm 2,132$ & $9,377 \pm 2,523$ & $9,761 \pm 2,379$ & $10,704 \pm 2,875$ & 1.67 & 0.178 \\
\hline \multirow{4}{*}{ Boys } & 3 & $9,215 \pm 392$ & $15,653 \pm 5,962$ & $13,235 \pm 3,064$ & $14,363 \pm 2,702$ & 2.21 & 0.098 \\
\hline & 4 & $10,881 \pm 4,494$ & $11,547 \pm 3,427$ & $13,047 \pm 2,485$ & $13,047 \pm 3,833$ & 3.60 & $0.015 *$ \\
\hline & 5 & $11,453 \pm 1,257$ & $11,876 \pm 3,485$ & $12,565 \pm 3,441$ & $12,834 \pm 2,895$ & 0.34 & 0.795 \\
\hline & 6 & $8,639^{\wedge}$ & $12,142 \pm 3,733$ & $12,223 \pm 3,578$ & $12,186 \pm 3,104$ & 0.35 & 0.25 \\
\hline
\end{tabular}

* significant difference at $\mathrm{p}<0.05$

$\wedge$ one participant, therefore no standard deviation. 


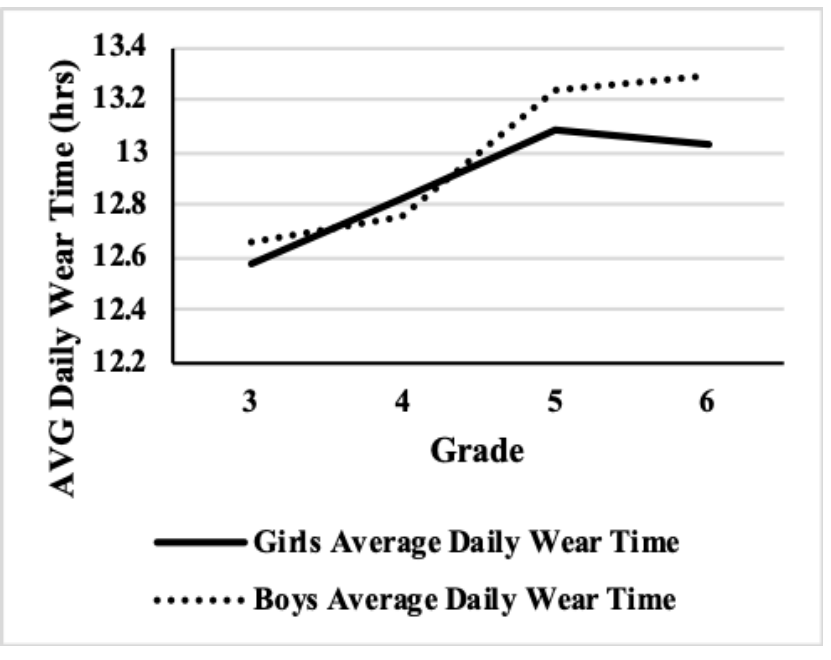

Figure 1. Average daily wear time for girls and boys by grade

Significant differences existed in average daily steps among the girls according to grade $(\mathrm{F}(3,461)=13.7$, $\mathrm{p}=0.000$ ). Post hoc analysis revealed the girls in grade 3 and 4 obtained significantly more steps than the girls in grades 5 and 6 (see Figure 2). A significant difference in average daily steps was also found for boys in grades 3,4 , 5 , and $6(\mathrm{~F}(3,383)=2.9, \mathrm{p}=0.033)$ with post hoc analyses indicating the boys in grade 3 took significantly more steps than boys in grades 4 and 6 (see Figure 2).

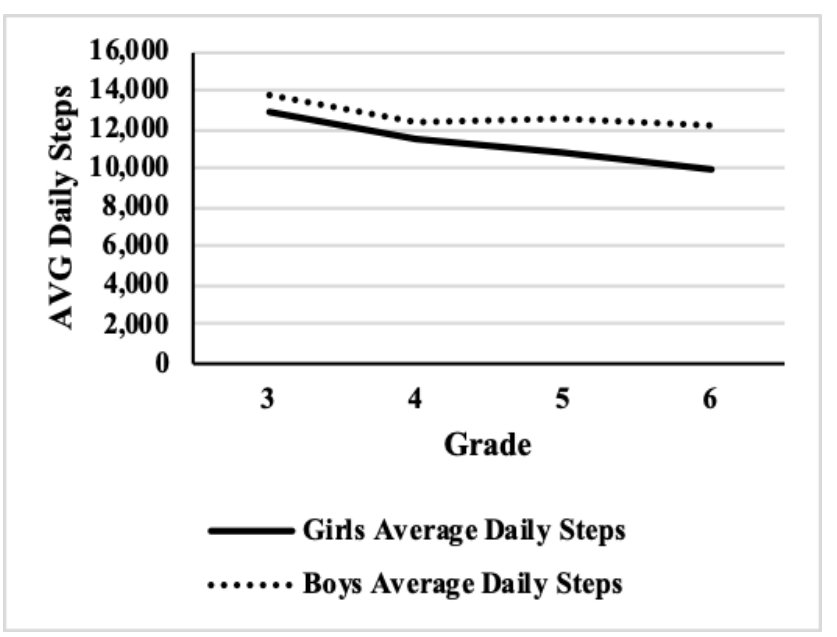

Figure 2. Average daily steps for girls and boys by grade

\section{Discussion}

The purpose of this study was to explore the average daily steps in children in grades 3 to 6 according to the number of valid pedometer days from a relatively large cross-sectional study. It is recommended that a minimum of three days of valid pedometer wear time be used as a guideline for including/excluding participants in further analyses (10). The results of this study indicated significant differences in the average daily steps obtained in children (boys and girls combined) in grades 3, 4, 5, and 6 who provided 1-2, 3-4, 5-6, and 7 days of pedometer data. More specifically, the children with 1-2 days of valid data had the least amount of steps, significantly less than the children with 7 days of valid data. Thus, excluding these children may lead to overestimating children's physical activity particularly since other researchers have suggested one and two days of valid pedometer data are sufficient in representing children's physical activity [14]. Further, the implications of excluding children from further analyses in various research projects due to insufficient days of pedometer data may limit attempts to better understand children and their physical activity. Although the ANOVA examining average steps per day according to sex and grade indicated a significant difference in the boys in grade 4, follow up post-hoc analyses showed no specific significant differences. Thus, the results from this study suggest inclusion of children with 1-2 days of valid pedometer data in future analyses.

Although not the primary focus of this study, it is worthy to note the overall average steps of the participants (i.e., 11,767) was slightly less than the recommended 12,000 steps/day for Canadian children (Colley et al., 2012). This finding is consistent with previous research on 1608 Canadian children aged 5 to 19 years in the Canadian Health Measures Survey, where an average of 11,220 steps/day was reported [3]. Further, it is recognized that among school-aged boys and girls, $10 \%$ and $6 \%$ respectively obtain the minimum average daily steps recommended [3]. In the present analyses, of those with 6-7 days of pedometer data (the number of days of pedometer data in the CHMS), 19\% obtained at least 12,000 steps/day.

The results of this study showed significant differences in average daily steps between grades, with the younger boys and girls obtaining more average daily steps. This finding is consistent with existing literature suggesting older children are less physically active than younger children [5,7]. Often the decrease in physical activity is reported to take place in the adolescent years, but these findings suggest that this decrease occurs earlier and is part of late childhood (i.e., in grades 5 and 6). A similar finding reported by Farooq et al suggests declines in physical activity are observed earlier than adolescence and then again in adolescence, with the decrease in adolescence not necessarily greater than in childhood [15].

Average daily pedometer wear time in boys and girls was significantly different between the higher and lower grades, with children in grades 5 and 6 wearing the pedometer significantly longer than the children in grades 3 and 4. This difference in wear time between the younger and older children may be partially a result of younger children sleeping longer. ParticipACTION reports that children aged 5-11 years sleep an average of 9.7 hours per night, while children aged 12-17 years obtain an average of 8.2 hours of sleep [4]. Although the older children report longer average pedometer wear times, this does not translate into more daily steps. The longer wear time and lower average daily steps with increasing grade suggests that the younger children are more physically active than the older children even though they wear the pedometer less. Although not further analyzed in this paper, there may in fact be an inverse relationship between pedometer wear time, and average daily physical activity which contradicts Ho et al. who found a positive relationship between pedometer wear and physical activity [16], though this association was only observed in girls.

A strength of this study was the relatively large sample size. Limitations include self-report of pedometer wear time as well as the inability to determine actual pedometer 
wear time. Future research regarding compliance to pedometer collection of physical activity data in children could employ alternative strategies where children are asked directly about their compliance and/or how they feel about wearing pedometers. Understanding children's compliance with data collection protocols could also help to better understand not only their level of physical activity, but also their motivations or lack thereof for physical activity. In other words, there could be differences in other physical activity related aspects not explored here, which may describe reasons for the difference in wear time compliance.

In conclusion, average daily steps and average wear time of elementary school-aged children who provided 1-2, $3-4,5-6$, or 7 days of pedometer data were compared in this study. Although significant differences were found in steps between the number of days of valid data, it is believed that children who provide 1-2 days of valid pedometer data should be included in further analyses when attempting to better understand children and their physical activity behaviors. When considering overall physical activity and wear time results, these analyses suggest that children who wear the pedometer less (i.e., 1-2 days) obtain fewer steps than the children who provide more days of physical activity data. If these findings are consistently found and these individuals are typically removed from analyses, we are missing an opportunity to learn more about physical activity in the samples studied. Overall, the results inform aspects of average wear time and average physical activity not commonly observed in the literature, and may be an important component of understanding and measuring children's physical activity.

\section{Acknowledgements}

This study was funded by the Royal Bank of Canada (RBC), the Public Health Agency of Canada, and Mitacs, and was delivered in partnership with ParticipACTION. The authors have no competing interests to report.

\section{References}

[1] Beets, MW, Patton, MM, Edwards, S. The accuracy of pedometer steps and time during walking in children. Med Sci Sports Exerc. 2005, 37(3). 513520 .
[2] Tremblay, MS, Carson, V, Chaput, J-P, et al. Canadian 24-hour movement guidelines for children and youth: An integration of physical activity, sedentary behavior, and sleep. Appl Physiol Nutr Me. 2016, 41. 311-327.

[3] Colley, RC, Janssen, I, Tremblay, MS. Daily step target to measure adherence to physical activity guidelines in children. Med Sci Sports Exerc. 2012, 44(5). 977-982.

[4] ParticipACTION. The Brain + Body Equation: Canadian kids need active bodies to build their best brains. The 2018 ParticipACTION Report Card on Physical Activity for Children and Youth. Toronto: ParticipACTION, 2018.

[5] Colley, RC, Garriguet, D, Janssen, I, Craig, CL, Clarke, J, Tremblay, MS. Physical activity in Canadian children and youth: accelerometer results from the 2007 to 2009 Canadian Health Measures Survey. Health Rep. 2011, 22(1). 15-23.

[6] Fukushima, N, Inoue, S, Hikihara, Y, et al. Pedometer-determined physical activity among youth in the Tokyo Metropolitan area: a cross-sectional study. BMC Public Health. 2016, 16. 1-12.

[7] Sherar, LB, Esliger, DW, Baxter-Jones, AD, Tremblay, MS. Age and gender differences in youth physical activity: Does physical maturity matter? Med Sci Sports Exerc. 2007, 39(5). 830-5.

[8] Corder, K, van Sluijs, EM, Ekelund, U, Jones, AP, Griffin, SJ. Changes in children's physical activity over 12 months: longitudinal results from the SPEEDY study. Pediatrics. 2010, 126(4). 926-935.

[9] Thompson, AM, Baxter-Jones, AD, Mirwald, RT, Bailey, DA. Comparison of physical activity in male and female children: Does maturation matter? Med Sci Sports Exerc. 2003, 35(10). 1684-1690.

[10] Tudor-Locke, C, Pangrazi, RP, Corbin, CB, et al. BMI-referenced standards for recommended pedometer determined steps/day in children. Prev. Med. 2004, 38(6). 857-864.

[11] Colley, R, Connor Gorber, S, Tremblay, MS. Quality control and data reduction procedures for accelerometry-derived measures of physical activity. Health Reports. 2010, 21(1). 1-8.

[12] Eisenmann, JC, Laurson, KR, Wickel, EE, Gentile, D, Walsh, D. Utility of pedometer step recommendations for predicting overweight in children. Int J Obes. 2007, 31. 1179-1182.

[13] Pabayo, R, Gauvin, L, Barnett, TA, Nikiema, B, Seguin, L. Sustained active transportation is associated with a favorable body mass index trajectory across early school years. Findings from the Quebec Longitudinal Study of Child Development birth cohort. Prev. Med. 2010, 50(Suppl. 1). 59-64.

[14] Craig, CL, Tudor-Locke, C, Cragg, S, Cameron, C. Process and treatment of pedometer data collection for youth: The Canadian physical activity levels among youth study. Med. Sci. Sports Exerc. 2010, 42(3). 430-5.

[15] Farooq, MA, Parkinson, KN, Adamson, AJ, et al. Timing of the decline in physical activity in childhood and adolescence: Gateshead Millennium Cohort Study. Br J Sports Med. 2018, 52. 1002-6.

[16] Ho, V, Simmons, RK, Ridgway, CL, et al. Is wearing a pedometer associated with higher physical activity in adolescents? Prev Med. 2013, 56(5). 273-7. 